\title{
Short Report: The Value of the Otorhinolaryngologic Exam in Correct Mucocutaneous Leishmaniasis Diagnosis
}

\author{
Viviane S. Boaventura, Jene G. S. de Oliveira, Jackson M. L. Costa, Fernanda O. Novais, Camila I. de Oliveira, \\ Manoel Barral-Netto, and Aldina Barral* \\ Centro de Pesquisas Gonçalo Moniz, Fundação Oswaldo Cruz (FIOCRUZ), Salvador, Brazil; Fundação Hospitalar do Acre (Fundhacre), \\ Rio Branco, Brazil; Universidade Federal da Bahia, Salvador, Brazil; Instituto de Investigação em Imunologia, Salvador, Brazil
}

\begin{abstract}
An increase in mucocutaneous leishmaniasis (ML) cases in northern (Brazil) motivated this study. In 44 ML patients with clinical diagnosis, only 13 parasitologically confirmed cases exhibited mucosal lesion suggestive of ML. Other conditions involving nasal manifestations are frequently confounded with ML. Therefore, otorhinolaryngologic examination is important in the clinical management of ML.
\end{abstract}

Tegumentary leishmaniasis (TL) remains a serious public health problem in several areas of the World, including Brazil. Mucosal leishmaniasis (ML) appears in about 3\% of cutaneous leishmaniasis (CL) patients in the Americas, and leads to destructive lesions in the nose, mouth, and larynx..$^{1,2}$ Mucosal leishmaniasis diagnosis remains difficult because clinical findings are nonpathognomonic and parasites are rarely found in mucosal lesions. ${ }^{3,4}$ The intradermal delayed-type hypersensitivity (DTH) skin test is highly sensitive and is usually required to confirm clinical suspicion. ${ }^{3,5}$ In parallel, histopathologic analysis or anti-Leishmania serology are used for diagnosis of ML. Considering that ML patients do not undergo spontaneous cure and present fatal outcome if left untreated and that ML treatment requires a longer drug regiment than $\mathrm{CL},{ }^{6-8} \mathrm{a}$ proper diagnosis of this particular clinical manifestation is of utmost importance.

In this report, we took advantage of an unexpected rise in the number of reported ML cases that occurred in Acre State (northern Brazil) from 2000 to 2002, to address the role of otorhynolaryngologic assessment in the diagnosis of ML. In this period, the incidence of TL (ML + CL) scaled from 141.9 to 223.7 cases $/ 100,000$ habitant. The ML cases represented $25 \%$ (197) of total TL (594) cases in 2000 and reached $28 \%$ (363) of total TL (1313) cases in 2002 (Secretaria de Saúde do Estado do Acre, Brazil, personal communication), both values above the previously documented $4 \%$ of total TL cases. ${ }^{1-3}$ Such an increase in ML frequency could be attributable to differences in virulence of the circulating Leishmania strain, ${ }^{9}$ or to incorrect or incomplete treatment of $\mathrm{CL}^{10}$ or to, for example, inadequate physical exam that could over diagnose ML.

To study the high proportion of mucosal involvement within TL cases in Acre, we performed a complete otorhinolaryngologic examination and, in parallel, we performed parasitologic and immunologic tests applied at the diagnosis of leishmaniasis in 44 patients with previous diagnosis of ML.

\section{THE STUDY}

Patients originated from three areas in Acre (Rio Branco, Sena Madureira, and Antimari) where the rate of ML was estimated as being $24.9 \%$ of TL cases (Secretaria de Saúde do Estado do Acre, Brazil, personal communication). Forty-four

\footnotetext{
*Address correspondence to Aldina Barral, Centro de Pesquisas Gonçalo Moniz/FIOCRUZ, Rua Waldemar Falcão, 121, Candeal, CEP 40296-710 Salvador, Bahia, Brazil. E-mail: abarral@bahia.fiocruz.br
}

patients (68\% men, $30 \pm 17$ years) with an ML diagnosis the previous year were submitted to a new evaluation incorporating an othorhinolaryngologic exam in 2002. Initial ML diagnosis was performed by a clinical physician in rural or urban health centers based on a nasal clinical complaint combined with one of the following: positive intradermal skin test; positive anti-Leishmania serology, or diagnosis of CL in the past (Table 1). Routine examination of mucosal surface by an otorhinolaryngologist had not been performed previously. For the present report, ML diagnosis was based on presence of lesions compatible with ML, a positive anti-Leishmania DTH skin test, positive anti-Leishmania serology, and the presence of Leishmania in biopsy samples detected by immunohistochemistry or by polymerase chain reaction (PCR).,11,12 Complete otorhinolaryngologic examination (anterior rhinoscopy, oropharynx exam, and a fiberoptic exam) was performed in all 44 patients. Complementary exams were performed if a characteristic ML lesion with edema, erosion, septal perfuration and/or granulomatous aspect was detected. Patients were informed of procedures and consented to participate in the study.

After otorhinolaryngologic evaluation, only 13 patients (29\%) presented mucosal lesion compatible with ML. Three patients within this group had been previously treated for ML and, accordingly, presented mucosal scars in the nasal septum. The remaining 10 patients presented active ML with ulceration, hyperemia, and granulomatous aspect, usually at the inferior turbinate and nasal septum perforation. Extensive lesions with pharynx and/or larynx involvement were detected in three patients. Mucosal complaints initiated from 2 months to 16 years of age before ear-nose-throat (ENT) exam. Leishmania infection was confirmed by PCR in all of the eight patients who consented to a biopsy procedure (Table 1 ). The remaining two patients with active mucosal lesion presented a positive DTH skin test in addition to a characteristic ML lesion. All 10 patients were treated with antimoniate-n-methylglucamine $(20 \mathrm{mg} / \mathrm{sb} / \mathrm{kg} / \mathrm{d})$ for $47 \pm 16$ days with clinical cure after 6 years follow-up time.

Nonetheless, ML diagnosis was not confirmed in $70 \%$ patients (31/44) present in the initial group. Otorhinolaryngologic examination did not detect scars, active ML, or other lesions of granulomatous aspect. Instead, clinical evaluation suggested other pathologies such as atrophic or allergic rhinitis, septum deviation, chronic sinusitis, and nasal polyps and biopsy procedures were not performed. Diagnoses were later confirmed by additional exams, such as skin prick test to allergens and face computed tomography (CT). ${ }^{13,14}$ 
TABLE 1

Clinical and laboratory data of ML and other mucosal disease patients*

\begin{tabular}{lcc}
\hline & \multicolumn{1}{c}{ Confirmed ML } & Other disease \\
\cline { 2 - 3 } & $N=8$ & $N=6 \dagger$ \\
\cline { 2 - 3 } & \multicolumn{2}{c}{ Positive (\%) } \\
\hline Clinical complaints & 100 & 100 \\
Previous CL & 75 & 83 \\
Serology & 75 & 66 \\
DTH & 87 & 100 \\
Histopathology & 75 & $\mathrm{NP}$ \\
PCR & 100 & $\mathrm{NP}$ \\
\hline *ML = mucosal leishmaniasis; $C \mathrm{CL}=$ cutaneous leishmaniasis; DTH = delayed-hypersensi- \\
tivity; PCR = polymerase chain reaction. \\
$\quad$ Biopsy not performed.
\end{tabular}

(

Although ML lesions were not detected in 31 patients, these presented clinical data suggestive of ML diagnosis. Thirteen patients $(42 \%)$ with erroneous ML diagnosis presented evidence of previous CL, as suggested by clinical history, the presence of scar, positive intradermal skin test and/or parasitologic confirmation. Unspecific nasal symptoms, such as epistaxis, itch, sneeze, nasal crust, secretion and/or obstruction, were detected in all 31 patients. Ten out of 31 (32\%) patients were submitted to DTH skin test and all of them presented a strong positive reaction to Leishmania antigen (Table 1). Based on initial ML diagnosis, 14/31 patients (45\%) initiated antimoniate-n-methyl-glucamine therapy and treatment was interrupted when ML diagnosis was not confirmed.

\section{CONCLUSIONS}

Our study presents strong evidence that ML diagnosis based only in clinical nasal symptoms and positivity in antiLeishmania immunologic tests, such as DTH or serology, leads to overestimation in the number of ML cases. Immunologic tests such as the Montenegro skin test remain positive for a long period of time and may lead to false diagnosis. ${ }^{1-3}$ Conversely, parasitologic diagnosis, albeit reliable, may face resistance from patients due to need of performing a biopsy. In the present series, all 44 patients that were initially diagnosed as ML cases were not, at the time of their first clinical examination, evaluated by an otorhinolaryngologist. A proper otorhinolaryngologic examination confirmed ML in only 13 of the 44 initially diagnosed patients. If mucosal examination were to be included in the initial diagnostic procedure, complementary exams would have eliminated 31 patients, because of the lack of mucosal lesion compatible with ML.

A positive immunologic exam associated to clinical complaints confounded the diagnostic procedure. Positive intradermal DTH skin test and serology are frequently detected among healthy endemic area residents. ${ }^{1}$ In this case, $83 \%$ of those individuals had CL previously, which explains their positive immunologic tests, and all of them presented symptoms that are not specific to ML, such as epistaxis, sneeze, and nasal obstruction.

Complementary tests were not necessary to exclude ML suspicion in those patients. However, they are important to confirm ML diagnosis if a characteristic mucosal lesion is detected. Certain granulomatous diseases such as paracoccidioidomycosis, leprosy, and syphilis present similar clinical aspects. ${ }^{3}$ In these cases, histopathologic analysis may rule out incorrect initial suspicion, but PCR is of utmost importance for definitive diagnosis. ${ }^{11,12}$ Furthermore, a positive DTH skin test may suggest this etiology but cannot be used to predict active disease in mucosal leishmaniasis because most individuals in endemic areas have been exposed to infected sand fly bites and may, therefore, present a positive reaction. Nonetheless, a negative result strongly suggests another diagnosis. ${ }^{2}$ We propose an algorithm for ML diagnosis: otorhinolaryngologic examination may be first performed if ML is suspected. Complementary exams such as intradermal DTH skin test, serology, and histopathology should be executed if a characteristic ML lesion is detected.

In the present context, incorrect ML diagnosis determined antimonial therapy for as long as 3 months. Indication to start antimonial therapy without ENT examination was inappropriate and, consequently, treated patients were submitted to unnecessary adverse side effects, penalizing the health system as well. Otorhinolaryngologic examination, as the first step in evaluation of suspect ML cases, may avoid incorrect ML diagnosis, which reached $70 \%$ in this study, preventing inappropriate treatment. The increased number of reported ML cases in Acre state was attributed to an incomplete clinical exam and overestimation of the value of complementary exams. Otorhinolaryngologic evaluation should be performed in all ML suspected cases before other diagnostic methods to avoid incorrect diagnosis and treatment.

Received December 15, 2008. Accepted for publication May 7, 2009.

Acknowledgments: We thank the clinical personnel at the Barral y Barral health post for assistance with patient recruitment and Ana Nilce Maia-Elkhoury from Fundação Nacional de Saúde (FUNASA).

Financial support: This study was funded by the Brazilian Ministry of Health. V. Boaventura and F. O. Novais were supported by a CAPES fellowship. J. M. Costa, C. I. de Oliveira, M. Barral-Netto, and A. Barral are senior investigators from CNPq.

Authors' addresses: Viviane Boaventura, Jackson Costa, Fernanda Novais, Camila I. de Oliveira, Manoel Barral-Netto, and Aldina Barral, Centro de Pesquisas Gonçalo Moniz/FIOCRUZ, Rua Waldemar Falcão, 121, Candeal, CEP 40296-710 Salvador, Bahia, Brazil, E-mails: voliveira@aluno.bahia.fiocruz.br, jcosta@bahia.fiocruz.br, novais@ aluno.fiocruz.br, camila@bahia.fiocruz.br, mbarral@bahia.fiocruz.br, and abarral@bahia.fiocruz.br. Jene de Oliveira, Fundação Hospital do Acre, BR 364, KM2, Distrito Industrial, 69900-000, Rio Brano, AC, Brazil, E-mail: junior.jene@uol.com.br.

\section{REFERENCES}

1. Marsden PD, 1986. Mucosal leishmaniasis ("espundia” Escomel, 1991). R Soc Trop Med Hyg 80: 859-876.

2. Barral A, Pedral-Sampaio D, Grimaldi G Jr, Momen H, McmahonPratt D, Jesus AR, Almeida R, Badaró R, Barral-Neto M, Carvalho EM, Johnson WD, 1991. Leishmaniasis in Bahia, Brazil: evidence that Leishmania amazonensis produces a wide spectrum of clinical disease. Am J Trop Med Hyg 44: 536-546.

3. Zajtchuk JT, Casler JD, Netto EM, Grogl M, Neafie RC, Hessel CR, De Magalhaes AV, Marsden PD, 1999. Mucosal leishmaniasis in Brazil. Laryngoscope 99: 925-939.

4. Herwaldt BL, 1999. Leishmaniasis. Lancet 354: 1191-1199.

5. Manzur A, Bari AE, 2006. Sensitivity of leishmanin skin test in patients of acute cutaneous leishmaniasis. Dermatol Online $J$ 12: 2 .

6. Marsden PD, Lessa HA, Oliveira MR, Romero GA, Marotti JG, Sampaio RN, Barral A, Carvalho EM, Cuba CC, Magalhães AV, Macêdo VO, 1998. Clinical observations of unresponsive mucosal leishmaniasis. Am J Trop Med Hyg 59: 543-545.

7. Grogl M, Thomasin TN, Franke ED, 1992. Drug resistence in leishmaniasis; its implication in systemic chemotherapy of cutaneous and mucocutaneous disease. Am J Trop Med Hyg 47: 117-126. 
8. Costa JML, Netto EM, Masden PD, 1986. Acute airway obstruction due to oedema of the larynx following antimony therapy in mucosal leishmaniasis. Rev Soc Bras Med Trop 19: 109.

9. da Silva ACT, Cupolillo E, Volpini AC, Almeida R, Romero GAS, 2006. Species diversity causing human cutaneous leishmaniasis in Rio Branco, state of Acre, Brazil. Trop Med Int Health 11: 1388-1398.

10. Llanos-Cuentas EA, Marsden PD, Cuba CC, Barreto AC, Campos M, 1984. Possible risk factors in development of mucosal lesions in leishmaniasis. Lancet 2: 295.

11. Oliveira CI, Báfica A, Oliveira F, Favali CB, Correa T, Freitas LAR, Nascimento E, Costa JM, Barral A, 2003. Clinical utility of polymerase chain reaction-based detection of Leishmania in the diagnosis of American cutaneous leishmaniasis. Clin Infect Dis 37: 149-153.

12. Oliveira JG, Novais FO, de Oliveira CI, da Cruz Junior AC, Campos LF, da Rocha AV, Boaventura V, Noronha A, Costa JM, 2005. Polymerase chain reaction (PCR) is highly sensitive for diagnosis of mucosal leishmaniasis. Acta Trop 945: $5-59$.

13. Szeinbach SL, Harpe SE, Williams PB, Elhefni H, 2008. Testing for allergic disease: parameters considered and test value. $B M C$ Fam Pract 9: 47

14. Younis RT, Anand VK, Davidson B, 2002. The role of computed tomography and magnetic resonance imaging in patients with sinusitis with complications. Laryngoscope 112: 224-229. 